\title{
AN ELEMENTARY PROOF OF WEYL'S LIMIT-CLASSIFICATION
}

\author{
J. DAS (neé CHAUDHURI)
}

(Received 24 April 1987)

Communicated by A. J. Pryde

\begin{abstract}
It is known [Herman Weyl, 1910] that every linear second-order differential expression $L$ (with real coefficients) is such that $L y=\lambda y(\operatorname{im} \lambda \neq 0)$ has at least one solution belonging to the class $\mathscr{L}^{2}=\mathscr{L}^{2}[0, \infty)$ of functions, the squares of whose moduli are Lebesgue-integrable on $[0, \infty)$. This celebrated result was later proved by E. C. Titchmarsh (1940-1944), using sophisticated analysis of bilinear transformations. The aim of the present note is to prove the same result once again, but using only elementary analysis and school geometry. The power of this method will be appreciated further when one realises the amount of simplifications that can be achieved by this method in case of higher order expressions. This part of the note of course will be taken up in a subsequent paper.
\end{abstract}

1980 Mathematics subject classification (Amer. Math. Soc.) (1985 Revision): 34 B 20.

Herman Weyl, in his classical paper of 1910, proved that every second-order ordinary differential equation of the form

$$
L[y]=-y^{(2)}(x)+q(x) y(x)=\lambda y(x)
$$

(where $y^{(r)}(x)=d^{r} y / d x^{r}, r=1,2, q(\cdot)$ is real-valued continuous over any bounded subinterval of $[0, \infty))$, has at least one solution $\psi \equiv \psi(x, \lambda)$ satisfying

$$
\int_{0}^{\infty}|\psi(x, \lambda)|^{2} d x<\infty
$$

whenever $\nu=\operatorname{im} \lambda \neq 0$. Such a solution $\psi$ is referred to as an $L^{2}$-solution of (1.1). If two independent solutions of (1.1) are $L^{2},(1.1)$ is said to be in the limit-circle case at infinity, while if only one independent solution is $L^{2}$, the

(C) 1989 Australian Mathematical Society $0263-6115 / 89 \$ A 2.00+0.00$ 
other is not $L^{2},(1.1)$ is said to be in the limit-point case at infinity. This is the celebrated limit classification of Weyl.

During 1940-1944, E. C. Titchmarsh [2] developed a really sophisticated theory based on the properties of bilinear transformations of the complex plane and established the above mentioned result. He associated the differential equation (1.1) with the boundary conditions

$$
\begin{aligned}
& y(0) \cos \alpha+y^{(1)}(0) \sin \alpha=0, \\
& y(b) \cos \beta+y^{(1)}(b) \sin \beta=0 .
\end{aligned}
$$

where $\alpha, \beta$ are arbitrary real numbers. Two linearly independent solutions $\theta=\theta(x) \equiv \theta(x, \lambda), \phi \equiv \phi(x) \equiv \phi(x, \lambda)$ of (1.1) are defined to satisfy the following:

$$
\begin{array}{ll}
\theta(0)=\cos \alpha, & \theta^{(1)}(0)=\sin \alpha, \\
\phi(0)=\sin \alpha, & \phi^{(1)}(0)=-\cos \alpha .
\end{array}
$$

Hence the general soltuion of (1.1) will be of the form $\theta+l(\lambda) \phi$ and if it is to satisfy the boundary condition (1.4) at $x=b$, we get

$$
\{\theta(b)+l(\lambda) \phi(b)\} \cos \beta+\left\{\theta^{(1)}(b)+l(\lambda) \phi^{(1)}(b)\right\} \sin \beta=0
$$

or

$$
l(\lambda)=-\frac{\theta(b) z+\theta^{(1)}(b)}{\phi(b) z+\phi^{(1)}(b)},
$$

where $z=\cot \beta$. In [2] Titchmarsh interpreted (1.8) as a bilinear transformation $T$ carrying the $z$-plane to the $l$-plane. As $\beta$ is real, by varying $\cot \beta$, we get the real line on the $z$-plane which, under the bilinear transformation $T$ is mapped onto a circle, $C_{b}$ say, on the $l$-plane. Using the fact that a bilinear transformation carries inverse points with respect to a circle into inverse points with respect to the corresponding image circle, Titchmarsh proved that, for any point $l=l(\lambda)$ inside the circle $C_{b}$,

$$
\int_{0}^{b}|\theta+l(\lambda) \phi|^{2} d x<-\frac{\operatorname{im} l(\lambda)}{\nu} .
$$

This is the vital inequality which proves that the circles $\left\{C_{b}\right\}_{b}$ form a monotonic decreasing set in the sense that if $b^{\prime}>b$ and $l(\lambda) \in C_{b^{\prime}}$, then $l(\lambda) \in C_{b}$ also. The idea of the present paper is to prove the inequality (1.9) directly, using elementary geometry only, without viewing (1.8) as a bilinear transformation. More precisely, it will be shown that (1.8) is actually the equation of the circle $C_{b}$ and that (1.8) can be exhibited as

$$
\int_{0}^{b}|\theta+l(\lambda) \phi|^{2} d x=-\frac{\operatorname{im} l(\lambda)}{\nu}
$$


so that the inequality (1.9) holds for any point $l(\lambda)$ inside $C_{b}$. It should be remarked here that this method is not only applicable to any higher even order differential equation, it avoids the whole lot of complications involved in dealing with the singular surfaces that are to be associated with higher order equations, as in Everitt [1].

Section 2 states a useful lemma of Titchmarsh with the deductions necessary for our purpose. Section 3 derives the equation of the circle $C_{b}$, while Section 4 gives the special form of the equation of the circle $C_{b}$. Finally Section 5 indicates that the method given here can be extended to higher even order differential equations.

\section{An important lemma}

The following Lemma of Titchmarsh ([2], page 23) is vital for our purpose:

LEMMA. If $F \equiv F(x, \lambda)$ is a solution of $L[y]=\lambda y$ and $G \equiv G(x, \lambda)$ is a solution of $L[y]=\lambda^{\prime} y$, then

$$
\left(\lambda^{\prime}-\lambda\right) \int_{a}^{b} F G d x=W_{a}(F, G)-W_{b}(F, G)
$$

where

$$
W_{x}(F, G)=F(x) G^{\prime}(x)-F^{\prime}(x) G(x)
$$

and $0 \leq a \leq b<\infty$.

The following special cases of the lemma can be deduced easily: $(\lambda=\mu+i \nu$, $\bar{\phi}=\phi(x, \bar{\lambda}), \bar{\theta}=\theta(x, \bar{\lambda}))$ :

$$
\begin{aligned}
& W_{b}(\phi, \bar{\phi})=2 i \nu \int_{0}^{b}|\phi|^{2} d x \\
& W_{b}(\phi, \bar{\theta})=1+2 i \nu \int_{0}^{b} \phi \bar{\theta} d x \\
& W_{b}(\theta, \bar{\theta})=2 i \nu \int_{0}^{b}|\theta|^{2} d x
\end{aligned}
$$




\section{The circle $C_{b}$ corresponding to the parameter in the boundary condition}

We write

$$
\begin{aligned}
& l(\lambda)=u+i v, \\
& \theta(b)=\theta(b, \lambda)=\xi(b, \lambda)+i \eta(b, \lambda)=\xi+i \eta, \\
& \phi(b)=\phi(b, \lambda)=\chi(b, \lambda)+i \tau(b, \lambda)=\chi+i \tau,
\end{aligned}
$$

where $u, v$ are real numbers and $\xi(b, \lambda), \eta(b, \lambda), \chi(b, \lambda), \tau(b, \lambda)$ are real, even though $\lambda$ is complex. Also we use the following notation:

$$
\begin{aligned}
& \theta^{(1)}(b)=\xi^{(1)}(b, \lambda)+i \eta^{(1)}(b, \lambda)=\xi^{\prime}+i \eta^{\prime}, \\
& \phi^{(1)}(b)=\chi^{(1)}(b, \lambda)+i \tau^{(1)}(b, \lambda)=\chi^{\prime}+i \tau^{\prime} .
\end{aligned}
$$

Then (1.7) gives, on equating real and imaginary parts,

$$
\begin{aligned}
& (\xi+u \chi-v \tau) \cos \beta+\left(\xi^{\prime}+u \chi^{\prime}-v \tau^{\prime}\right) \sin \beta=0 \\
& (\eta+u \tau+v \chi) \cos \beta+\left(\eta^{\prime}+u \tau^{\prime}+v \chi^{\prime}\right) \sin \beta=0
\end{aligned}
$$

whence, eliminating $\beta$, we get the locus of the point $l=l(\lambda)=u+i v$ as

$$
\begin{aligned}
S_{b}(u, v)= & \left(u^{2}+v^{2}\right)\left(\chi \tau^{\prime}-\chi^{\prime} \tau\right)+u\left(\chi \eta^{\prime}+\xi \tau^{\prime}-\chi^{\prime} \eta-\xi^{\prime} \tau\right) \\
& +v\left(\chi^{\prime} \xi-\eta^{\prime} \tau+\eta \tau^{\prime}-\chi \xi^{\prime}\right)+\xi \eta^{\prime}-\xi^{\prime} \eta=0
\end{aligned}
$$

Next we note that

$$
W_{b}(\phi, \bar{\phi})=\phi(b) \bar{\phi}^{(1)}(b)-\phi^{(1)}(b) \bar{\phi}(b)=-2 i\left(\chi \tau^{\prime}-\chi^{\prime} \tau\right)
$$

so that

$$
\chi \tau^{\prime}-\chi^{\prime} \tau=-\nu \int_{0}^{b}|\phi|^{2} d x
$$

Since $\phi$ is a non-trivial solution of (1.1), we have $\chi \tau^{\prime}-\chi^{\prime} \tau \neq 0$ unless $\nu=0$. Thus, as $\beta$ varies, the point $l=l(\lambda)=u+i v$ describes a circle, $C_{b}$ say, on the $(u, v)$-plane and the equation of $C_{b}$ is given by

$$
S_{b}(u, v)=0 .
$$

4. The equation of the circle $C_{b}$ and its limit as $b \rightarrow \infty$

In this section we establish that

$$
S_{b}(u, v) \equiv-\nu \int_{0}^{b}|\theta+l \phi|^{2} d x-\operatorname{im} l(\lambda)=0 .
$$


ProOF. It is easy to see, using (2.3)-(2.5), that

$$
\begin{aligned}
\int_{0}^{b}|\theta+l \phi|^{2} d x= & \int_{0}^{b}|\theta|^{2} d x+l \int_{0}^{b} \phi \bar{\theta} d x+\bar{l} \int_{0}^{b} \bar{\phi} \theta d x+|l|^{2} \int_{0}^{b}|\phi|^{2} d x \\
= & \left(u^{2}+v^{2}\right) \frac{W_{b}(\phi, \bar{\phi})}{2 i \nu}+\frac{u}{2 i \nu}\left\{W_{b}(\phi, \bar{\theta})-W_{b}(\phi, \theta)\right\} \\
& \left.+\frac{v}{2 \nu}\left\{W_{b}(\phi, \bar{\theta})+W_{b}(\bar{\phi}, \theta)\right\}-W_{b}(\bar{\phi}, \theta)\right\}-\frac{v}{\nu}+\frac{W_{b}(\theta, \bar{\theta})}{2 i \nu}
\end{aligned}
$$

Using the definitions of $W_{x}(F, G)$, we can immediately deduce that

$$
\begin{aligned}
& W_{b}(\phi, \bar{\theta})-W_{b}(\bar{\phi}, \theta)=-2 i\left(\chi \eta^{\prime}-\chi^{\prime} \eta+\xi \tau^{\prime}-\xi^{\prime} \tau\right) \\
& W_{b}(\phi, \bar{\theta})+W_{b}(\bar{\phi}, \theta)=-2\left(\chi^{\prime} \xi-\chi \xi^{\prime}+\eta \tau^{\prime}-\eta^{\prime} \tau\right) \\
& W_{b}(\theta, \bar{\theta})=-2 i\left(\xi \eta^{\prime}-\xi^{\prime} \eta\right)
\end{aligned}
$$

From (4.2), (3.8), (4.3)-(4.5), it is then easy to prove (4.1). Now, we apply elementary geometry only, to infer that a point $u+i v$ will lie inside the circle $C_{b}$, given by $S_{b}(u, v)=0$, if $S_{b}(u, v)<0$ when $\chi \tau^{\prime}-\chi^{\prime} \tau>0$ (that is, $\nu<0$ ) and if $S_{b}(u, v)>0$ when $\chi \tau^{\prime}-\chi^{\prime} \tau<0$ (that is, $\nu>0$ ); that is, if $\int_{0}^{b}|\theta+l \phi|^{2}+\operatorname{im} l / \nu<0$ for all $\nu \neq 0$, whether positive or negative.

\section{Remarks}

The method adopted in this paper does not involve the coefficients of the differential equation explicitly. Hence the differential equation can be replaced by

$$
M[y]=-\left(p y^{(1)}\right)^{(1)}+q y=\lambda y
$$

where $p$ is real-valued and continuous on $[0, \infty)$ and $p(x) \neq 0$ for and $x \in[0, \infty)$.

The method of this proof for the limit-classification can be used in the case of higher even order differential equations. The increase in the order of the differential equation will increase the dimension of the surface $S_{b}(u, v)=0$ and hence matrix techniques must be used. These techniques will be developed in a subsequent paper, where it will be shown how this method nicely avoids all the complications involved in determining the monotonic nature of the singular surfaces. 


\section{References}

[1] W. N. Everitt, 'Fourth-order singular differential equations,' Math. Ann. 149 (1963), 32040.

[2] E. C. Titchmarsh, Eigenfunction expansions associated with second order differential equations,' Part I (2nd ed., Clarendon Press, 1962).

[3] E. C. Titchmarsh, 'An extension of the Sturm-Liouville expansion,' Quart. J. Math. Oxford Ser. 15 (1944), 40-48.

[4] H. Weyl, 'Über gewöhnliche Differentialgleichungen mit Singularitäten und zugehörigen Entwicklungen willkürlicher Funktionen', Math. Ann. 68 (1910), 222-269.

Department of Pure Mathematics

Calcutta University

35, Ballygunge Circular Road

Calcutta - 700019

India 